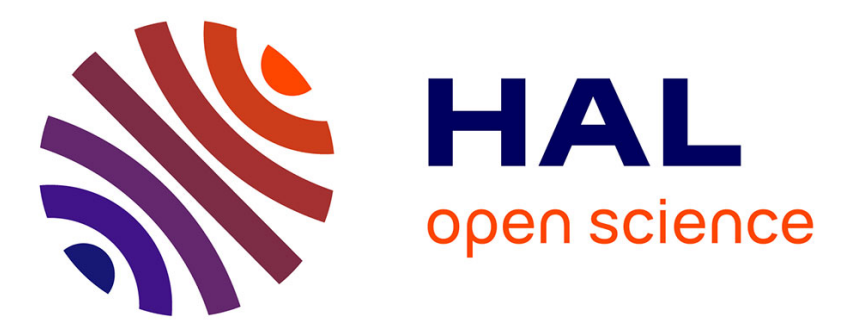

\title{
Resonance Suppression in Multi-Degree-of-Freedom Rotating Flexible Structures Using Order-Tuned Absorbers
}

\author{
Serif Gozen, Brian Olson, Steven Shaw, Christophe Pierre
}

\section{To cite this version:}

Serif Gozen, Brian Olson, Steven Shaw, Christophe Pierre. Resonance Suppression in Multi-Degreeof-Freedom Rotating Flexible Structures Using Order-Tuned Absorbers. Journal of Vibration and Acoustics, 2012, 134 (6), pp.061016. 10.1115/1.4007564 . hal-02434069

\section{HAL Id: hal-02434069 \\ https://hal.science/hal-02434069}

Submitted on 10 Jan 2020

HAL is a multi-disciplinary open access archive for the deposit and dissemination of scientific research documents, whether they are published or not. The documents may come from teaching and research institutions in France or abroad, or from public or private research centers.
L'archive ouverte pluridisciplinaire HAL, est destinée au dépôt et à la diffusion de documents scientifiques de niveau recherche, publiés ou non, émanant des établissements d'enseignement et de recherche français ou étrangers, des laboratoires publics ou privés. 


\section{RESONANCE SUPPRESSION IN MULTI-DOF ROTATING FLEXIBLE STRUCTURES USING ORDER-TUNED ABSORBERS}

\author{
Serif Gozen \\ Department of Mechanical Engineering \\ McGill University \\ Montreal, QC CANADA \\ Email: serif.gozen@mcgill.ca \\ Steven W. Shaw \\ Department of Mechanical Engineering \\ Michigan State University \\ East Lansing, MI 48824-1226 USA \\ Email: shawsw@egr.msu.edu
}

\author{
Brian J. Olson* \\ Air and Missile Defense Department \\ The Johns Hopkins University Applied Physics Laboratory \\ Laurel, MD 20723-6099 USA \\ Email: brian.olson@jhuapl.edu \\ Christophe Pierre \\ Department of Mechanical Engineering \\ McGill University \\ Montreal, QC CANADA \\ Email: christophe.pierre@mcgill.ca
}

\begin{abstract}
This paper considers the dynamic response and order-tuning of vibration absorbers fitted to a rotating flexible structure under traveling wave (TW) engine order excitation. Of specific interest is the extension of previous results on the so-called no-resonance zone, that is, a region in linear tuning parameter space in which the coupled structure/absorber system does not experience resonance over all rotation speeds. The no-resonance feature was shown to exist for cyclic rotating structures with one structural and one absorber degree-of-freedom (DOF) per sector. This work uses a higher-fidelity structural model to investigate the effects of higher modes on the cyclically-coupled system. It is shown that the no-resonance zone is replaced by a resonancesuppression zone in which one structural mode is suppressed, but higher-order resonances still exist with the addition of the absorbers. The results are general, in the sense that one vibration mode can be eliminated using a set of identically-tuned absorbers on a rotating structure with arbitrarily many DOFs per sector.
\end{abstract}

\footnotetext{
*Address all correspondence to this author.
}

\section{INTRODUCTION}

Many rotating flexible structures consist of an array of interconnected constituent parts whose geometry and structural properties are rotationally periodic (i.e., cyclically symmetric). In a bladed disk assembly, for example, the fundamental substructure is one blade plus the corresponding segment of the disk, which is collectively referred to as a sector. During steady operation these systems rotate at a constant speed and are subjected to TW dynamic loading, or engine order (e.o.) excitation, which is characterized by excitation frequencies that are proportional to the mean rotational speed of the rotor. Such excitations result in component vibrations that can lead to high cycle fatigue failure, noise, reduced performance, and other undesirable effects. Order-tuned vibration absorbers exploit the centrifugal field from rotation of the primary system and are thus ideally-suited to address component vibration in rotating flexible structures. They essentially consist of masses that ride along designer-specified paths relative to the primary system. When properly tuned, these absorbers effectively counteract fluctuating loads applied to the primary system over all rotation speeds [1].

The dynamic performance, characteristics and features of 
order-tuned absorbers are well-understood in typical situations, and there are numerous examples of their implementation. They have been investigated by Shaw et al. [2-6] and others for torsional vibration reduction in rotating systems. Applications include light aircraft engines [7], helicopter rotors [8], diesel camshafts [9], and advanced technology automotive engines [10].

In previous works Olson et al. [11-15] investigated the performance of order-tuned vibration absorbers applied to lumpedparameter models of a cyclically-coupled bladed disk assembly under engine order excitation. In these models, the sector dynamics are captured by two DOFs: one for the blade and one for the absorber. A key finding from the linearized coupled system is the existence of the so-called "no-resonance zone" in linear tuning parameter space. It was shown that there exists a range of absorber tuning values, close to the ideal tuning that exactly matches the excitation order, for which there are no resonances over the entire range of rotation speeds. This result was first reported by Shaw et al. [16] for a model consisting of a single, isolated sector (i.e., one blade and one absorber attached to a rotating disk). Olson et al. showed that the no-resonance zone persists in the (coupled) multi-sector linear system [11-13] and that it qualitatively persists when weak nonlinearities are taken into account via the absorber paths $[12,14,15]$. Existence of the no-resonance zone allows for the possibility of robustly-tuned absorbers that can function effectively in the presence of model and parameter uncertainties.

This paper investigates if and how the no-resonance zone persists when higher-fidelity models are used to describe the bladed disk system. It will suffice to employ a 3-DOF sector model that consists of two DOFs for each blade/disk and one for the attendant absorber. The no-resonance zone is shown to persist in a restricted sense: one structural mode can be eliminated, but higher mode resonances still exist at some rotor speed for any absorber tuning. These results generically hold for models with many blade/disk DOFs because of the nature of dynamic coupling between the absorber and blade dynamics, which results in characteristic eigenvalue veering as the rotor speed is varied $[17,18]$. Suppression of multiple resonances may be achieved, for example, by implementing multiple sets of absorbers. This is left for future work.

The paper is organized as follows. A higher-fidelity model of a rotating bladed disk assembly under engine order excitation is developed in Section 2, where each blade is fitted with an order-tuned vibration absorber. Section 3 introduces a tuning strategy in which the absorber tuning order is set relative to the order of the TW excitation. Features of the forced response are discussed in Section 4, which contains the main results of this work. The paper closes in Section 5 with a discussion and suggestions for future work.

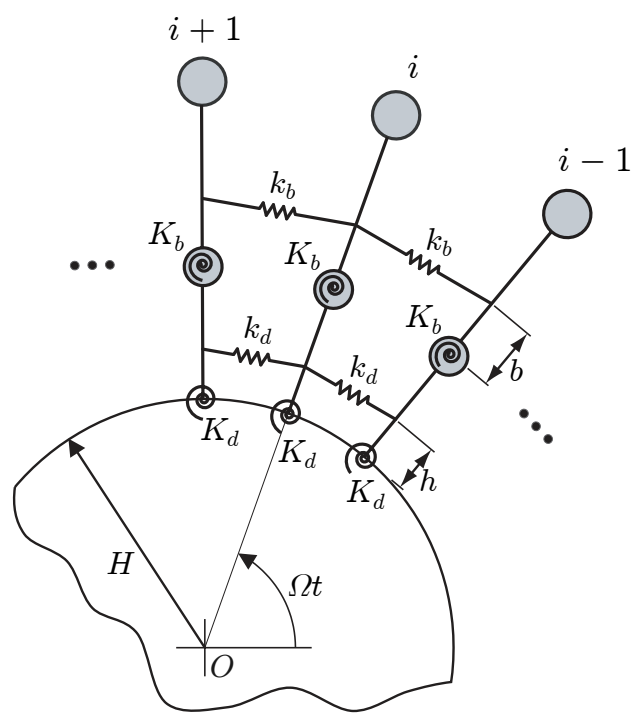

(a) Model of Bladed Disk Assembly

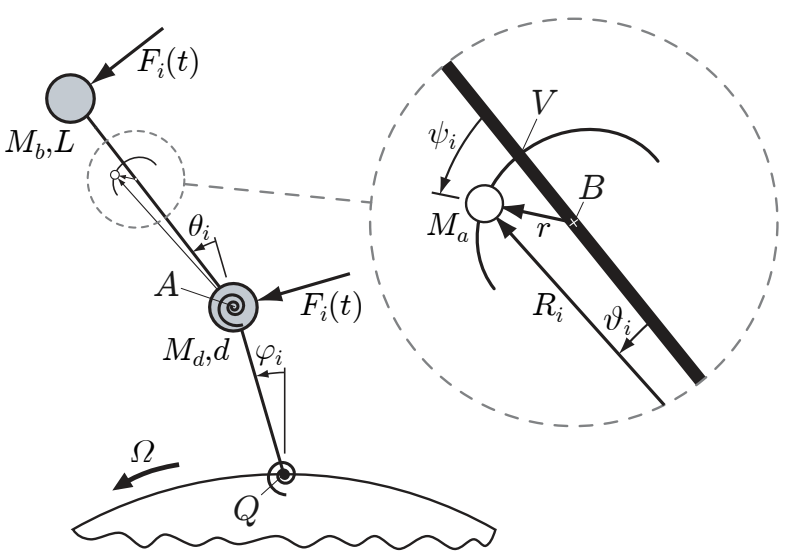

(b) Sector Model

Figure 1. Lumped-Parameter Model.

\section{SYSTEM MODEL}

A lumped-parameter model of the rotating bladed disk assembly is shown in Fig. $1 a$. It consists of a cyclic array of $N$ identical, identically-coupled sectors (Fig. 1b), each with one blade and one absorber plus a corresponding segment of the disk. The disk component of each sector is composed of a rigid part of radius $H$, which rotates at a constant speed $\Omega$ about a fixed axis through $O$. This component is referred to as the rotor. A second flexible portion vibrates about a point $Q$ at the periphery of the rigid rotor, which is modeled by a pendulum of length $d$ and mass $M_{d}$ and is referred to as the disk. The blade dynamics are captured by a second pendulum of length $L$ and mass $M_{b}$ that is attached to the disk pendulum at the vertex $A$. There are $N$ such double pendulum systems, which are uniformly distributed 
along the circumference of the rotor. The sector model shown in Fig. $1 b$ could also represent a 2-DOF blade model attached to a rigid disk/rotor. The key feature is multiple DOFs per sector, in this case two, which is sufficient to capture the effects ordertuned absorbers when there are arbitrarily many disk/blade DOFs per sector.

The flexural stiffness associated with the $i^{\text {th }}$ blade and disk is modeled using linear torsional springs of stiffness $K_{b}$ and $K_{d}$, respectively. Adjacent blades (resp. disk portions) are coupled via translational springs with stiffness $k_{b}$ (resp. $k_{d}$ ) at a distance $b$ (resp. $h$ ) relative to their attachment points to the disk (resp. rotor). It is assumed that the springs are unstressed when the disks and blades are in a purely radial configuration, that is, when $\varphi_{i}=\theta_{i}=0$ for each $i \in \mathcal{N}=\{1,2, \ldots, N\}{ }^{1}$ Each stiffness element is paired with a corresponding linear viscous damper (not shown in Fig. 1a) to capture the effects of dissipation. The disk and inter-disk (resp. blade and inter-blade) damping is modeled by linear torsional and translational dampers with constants $C_{d}$ and $c_{d}$ (resp. $C_{b}$ and $c_{b}$ ). It is convenient to model the absorber damping with an effective torsional damper with constant $C_{a}$ that acts at point $B$ (attachment point of absorber pendulum).

The blades are fitted with identical vibration absorbers, which generally consist of particle masses $M_{a}$ that ride on userspecified paths. Each absorber path is described by a vector of length $R_{i}\left(S_{i}\right)=R_{i}\left(-S_{i}\right)$ relative to the outermost blade pendulums, where $S_{i}$ is the path arc length relative to its vertex $V$. Polchi [19] derived the governing EOM for the system shown in Fig. $1 a$ fitted with the general-path absorbers using the path formulation described by Olson et al. [12, 14, 15]. However, for the small-amplitude linearized model to be developed, the absorbers can be regarded as simple pendulums with length $r$ and mass $M_{a}$ that are attached a distance $\alpha L$ along the outermost blade pendulum relative to point $A$. Their linearized dynamics are described by the relative angular coordinates $\psi_{i}=S_{i} / r$. Thus, each sector consists of a double-pendulum disk/blade model and a simple-pendulum (circular path) absorber attached to the outermost double-pendulim link.

The $i^{\text {th }}$ disk/blade double pendulums are harmonically forced in the transverse sense by e.o. excitation of order $n$ such that the cyclic system is circumferentially-forced by a TW. The e.o. excitation is modeled by $[12,20]$

$$
F_{i}(t)=F_{0} e^{j \phi_{i}} e^{j n \Omega t}
$$

where $\phi_{i}=2 \pi \frac{n}{N}(i-1)$ is the inter-sector phase angle, $F_{0}$ is the excitation strength, and $j=\sqrt{-1}$. The e.o. is restricted such that $n \in \mathcal{N}$, which includes all practically relevant situations [12].

The equations of motion (EOM) are formulated using Lagrange's method, linearized for small disk/blade and absorber

\footnotetext{
${ }^{1}$ Throughout the remainder of this work it is understood that variables and equations with subscripts $i$ are defined for each $i \in \mathcal{N}$.
}

Table 1. Selected list of dimensionless parameters.

\begin{tabular}{|c|c|}
\hline Parameters & Description \\
\hline$\delta=H / d$ & Radius of the rigid rotor disk \\
\hline$\rho=L / d$ & Length of blade pendulum \\
\hline$\alpha$ & Distance between points $A$ and $B$ \\
\hline$\gamma=r / d$ & Length of absorber pendulum \\
\hline$\mu_{a}=M_{a} / M_{d}$ & Absorber mass \\
\hline$\mu_{b}=M_{b} / M_{d}$ & Blade mass \\
\hline$f_{\varphi}=F_{0}(L+d) / K_{d}$ & Strength of e.o. excitation ( $\varphi_{i}$-dynamics) \\
\hline$f_{\theta}=F_{0} L / K_{d}$ & Strength of e.o. excitation ( $\theta$-dynamics) \\
\hline$\lambda=\sqrt{\frac{K_{b}}{K_{d}}}$ & Square of the blade torsional stiffness \\
\hline$v_{a}=\sqrt{\frac{\left(k_{d} h^{2}+k_{b} d^{2}\right)}{K_{d}}}$ & Stiffness coupling between sectors \\
\hline$v_{b}=\sqrt{\frac{k_{b} d b}{K_{d}}}$ & Stiffness coupling between sectors \\
\hline$v_{c}=\sqrt{\frac{k_{b} b^{2}}{K_{d}}}$ & Stiffness coupling between sectors \\
\hline$\xi_{a c}=\frac{1}{d^{2}} \frac{c_{d} h^{2}+c_{b} d^{2}}{\sqrt{K_{d} M_{d} / d^{2}}}$ & Damping coupling between sectors \\
\hline$\xi_{b c}=\frac{1}{d^{2}} \frac{c_{b} d b}{\sqrt{K_{d} M_{d} / d^{2}}}$ & Damping coupling between sectors \\
\hline$\xi_{c c}=\frac{1}{d^{2}} \frac{c_{b} b^{2}}{\sqrt{K_{d} M_{d} / d^{2}}}$ & Damping coupling between sectors \\
\hline$\xi_{a}=\frac{1}{d^{2}} \frac{C_{a}}{\sqrt{K_{d} M_{d} / d^{2}}}$ & Absorber torsional damping constant \\
\hline$\xi_{b}=\frac{1}{d^{2}} \frac{C_{b}}{\sqrt{K_{d} M_{d} / d^{2}}}$ & Blade torsional damping constant \\
\hline$\xi_{d}=\frac{1}{d^{2}} \frac{C_{d}}{\sqrt{K_{d} M_{d} / d^{2}}}$ & Disk torsional damping constant \\
\hline$\sigma=\Omega / \omega_{0}$ & Angular speed of the rotor \\
\hline
\end{tabular}

motions, and made dimensionless according to the nondimensional parameters defined in Table 1 . Time is rescaled by $\tau=\omega_{0} t$, where $\omega_{0}=\sqrt{K_{d} / M_{d} d^{2}}$ is the undamped natural frequency of a single isolated disk DOF. In matrix-vector form the sector EOM are given by

$$
\begin{aligned}
\mathbf{M z} \mathbf{z}_{i}^{\prime \prime}+\mathbf{C} \mathbf{z}_{i}^{\prime} & +\mathbf{K} \mathbf{z}_{i}+\mathbf{C}_{c}\left(-\mathbf{z}_{i-1}^{\prime}+2 \mathbf{z}_{i}^{\prime}-\mathbf{z}_{i+1}^{\prime}\right) \\
& +\mathbf{K}_{c}\left(-\mathbf{z}_{i-1}+2 \mathbf{z}_{i}-\mathbf{z}_{i+1}\right)=\mathbf{f e}^{j \phi_{i}} \mathrm{e}^{j n \sigma \tau}
\end{aligned}
$$

where the elements of the $3 \times 3$ sector mass, damping, and stiffness matrices $(\mathbf{M}, \mathbf{C}, \mathbf{K})$ are defined in Table 2, the vector $\mathbf{z}_{i}=$ $\left(\varphi_{i}, \theta_{i}, \psi_{i}\right)^{T}$ captures the sector dynamics, and $(\cdot)^{\prime}=d(\cdot) / d \tau$ denotes differentiation with respect to dimensionless time. Intersector coupling is captured by the matrices

$$
\mathbf{C}_{c}=\left[\begin{array}{ccc}
\xi_{a c} & \xi_{b c} & 0 \\
\xi_{b c} & \xi_{c c} & 0 \\
0 & 0 & 0
\end{array}\right], \quad \mathbf{K}_{c}=\left[\begin{array}{ccc}
\mathrm{v}_{a}^{2} & \mathrm{v}_{b}^{2} & 0 \\
\mathrm{v}_{b}^{2} & \mathrm{v}_{c}^{2} & 0 \\
0 & 0 & 0
\end{array}\right]
$$


Table 2. Elements of the sector mass, damping, and stiffness matrices.

\begin{tabular}{lll}
\hline$M_{11}=\mu_{b}\left(\rho^{2}+1\right)+\mu_{a}(\alpha+\gamma+1)^{2}+1$ & $C_{11}=\xi_{d}$ & $K_{11}=\delta\left(\mu_{b}(\rho+1)+\mu_{a}(\alpha+\gamma+1)+1\right) \sigma^{2}+1$ \\
$M_{12}=\mu_{b} \rho(\rho+1)+\mu_{a}(\alpha+\gamma)(\alpha+\gamma+1)$ & $C_{12}=-\xi_{b}$ & $K_{12}=\delta\left(\mu_{b} \rho+\mu_{a}(\alpha+\gamma)\right) \sigma^{2}$ \\
$M_{13}=\mu_{a} \gamma(\alpha+\gamma+1)$ & $C_{13}=-\xi_{a}$ & $K_{13}=\mu_{a} \gamma \delta \sigma^{2}$ \\
$M_{21}=M_{12}$ & $C_{21}=0$ & $K_{21}=K_{12}$ \\
$M_{22}=\mu_{b} \rho^{2}+\mu_{a}(\alpha+\gamma)^{2}$ & $C_{22}=\xi_{b}$ & $K_{22}=(\delta+1)\left(\mu_{b} \rho+\mu_{a}(\alpha+\gamma)\right) \sigma^{2}+\lambda^{2}$ \\
$M_{23}=\mu_{a} \gamma(\alpha+\gamma)$ & $C_{23}=-\xi_{a}$ & $K_{23}=\mu_{a} \gamma(\delta+1) \sigma^{2}$ \\
$M_{31}=M_{13}$ & $C_{31}=0$ & $K_{31}=K_{13}$ \\
$M_{32}=M_{23}$ & $C_{32}=0$ & $K_{32}=K_{23}$ \\
$M_{33}=\mu_{a} \gamma^{2}$ & $C_{33}=\xi_{a}$ & $K_{33}=\mu_{a} \gamma(\alpha+\delta+1) \sigma^{2}$ \\
\hline
\end{tabular}

and the sector forcing vector is given by $\mathbf{f}=\left(f_{\varphi}, f_{\theta}, 0\right)^{T}$, where the dimensionless entries are defined in Table 1 . The $3 N$ linearized EOM defined by Eq. (2) serve as the basis for the analysis that follows.

\section{ABSORBER TUNING}

The linear absorber tuning order follows from the special case when the disks and blades are locked in their zero positions relative to the spinning rotor $[11,12]$. This leads to a system of identical, dynamically isolated and unforced singleDOF absorbers that oscillate freely under the influence of centrifugal effects. Their dynamics follow from Eq. (2) by setting $\varphi_{i}=\varphi_{i}^{\prime}=\varphi_{i}^{\prime \prime}=0$ and $\theta_{i}=\theta_{i}^{\prime}=\theta_{i}^{\prime \prime}=0$. Then

$$
\mu_{a} \gamma^{2} \psi_{i}^{\prime \prime}+\xi_{a} \psi_{i}^{\prime}+\mu_{a} \gamma(\alpha+\delta+1) \sigma^{2} \psi_{i}=0
$$

where the undamped natural frequencies are given by

$$
\bar{\omega}_{33} \equiv \frac{\omega_{33}}{\omega_{0}}=\sqrt{\frac{\alpha+\delta+1}{\gamma}} \sigma .
$$

Physically, each absorber acts like a centrifugally-driven pendulum of length $\gamma$ whose pivot point is located a distance $\alpha+\delta+1$ from the rotor axis $O$. The base accelerates towards the center or rotation at a constant rate $(\alpha+\delta+1) \sigma^{2}$, which is the effective gravity of the pendulum. This results in a centrifugal restoring force that scales with $\sigma^{2}$ and an undamped natural frequency that scales with $\sigma$ according to the constant

$$
\tilde{n}=\sqrt{\frac{\alpha+\delta+1}{\gamma}}
$$

which is defined to be the absorber tuning order. Because the isolated absorber eigenfrequencies $\bar{\omega}_{33}$ and the e.o. excitation frequency scale directly with the rotor speed, the absorbers are tuned to a given order of the excitation as opposed to a particular frequency [21].

Absorber tuning refers to a choice of absorber geometric parameters, which are selected to attenuate the blade responses over a range of operating speeds, particularly near resonance. This is done by choosing a value for $\tilde{n}$ and then selecting the dimensionless parameters $\alpha, \delta$, and $\gamma$ to satisfy Eq. (6). It is shown that there exists an ideal absorber tuning that results in suppression of one of the blade resonances, where the attendant absorber amplitudes depend primarily on their mass and placement along the blade lengths and the level of the applied loads. Ideal tuning is defined by

$$
\tilde{n}=n
$$

in which case the isolated absorber natural frequency $\bar{\omega}_{33}$ identically matches that of the excitation. Exact tuning is effective for all rotation speeds, but it is susceptible to uncertainties of the model or absorber parameters due to in-service wear, environmental effects, or the effects of tolerances. To account for such effects, and to allow for intentionally detuned designs, we let

$$
\tilde{n}=n(1+\beta),
$$

where $\beta$ is a detuning parameter. Ideal linear tuning corresponds to $\beta=0$ while undertuning (resp. overtuning) corresponds to $\beta<0$ (resp. $\beta>0$ ). One of the main goals of absorber design is to select $\beta$ to achieve robust vibration reduction of the primary system.

\section{FEATURES OF THE FORCED RESPONSE}

This section reports the main results of the paper. The linearized EOM are analyzed to determine the effects of absorber tuning on the steady-state TW response to TW e.o. excitation. 
The special case for which the absorbers are locked at their vertices is analyzed in Section 4.1. These results, which are typical for a cyclic system with two DOF per sector, are used as a baseline against which the absorber performance is assessed. The full model, in which all three sector DOF are free to move, is considered in Section 4.2. Of particular interest are the effects of varying the absorber tuning and whether or not the no-resonance zone reported by Olson et al. [11-13] exists for this higher fidelity model.

The forced response can be obtained by assembling the sector models defined by Eq. (2) into a single matrix EOM and proceeding in the usual way [22]. For large $N$, however, it is desirable to simplify the analysis by exploiting the system (cyclic) symmetry, as it is done by Olson et al. [11-13] and others [23]. If $P$ is the number of DOF in each sector $(P=3$ for the model shown in Fig. 1), the $P N \times P N$ system mass, damping, and stiffness matrices are block circulant with $P \times P$ blocks. The $P N$-DOF model can be block decoupled to a set of $N, P$ DOF modal systems via a unitary transformation involving the complex Fourier matrix [24-26]. The $P N$ natural frequencies are preserved under the transformation and can be obtained in sets of $P$ from the $N$ decoupled modal EOM. The forced response follows accordingly, and allows for the full system response to be determined by considering a set of uncoupled three DOF models.

\subsection{Response with the Absorbers Locked}

If the absorbers are locked relative to the rotating blades, then $\psi_{i}=\psi_{i}^{\prime}=\psi_{i}^{\prime \prime}=0$ and each sector model has two DOFs. Figure $2 a$ shows the $2 N$ natural frequency loci in a Campbell diagram and the corresponding disk/blade amplitude frequency response $\left(\left|\varphi_{i}\right|,\left|\theta_{i}\right|\right)$ for a representative model with $N=10$ sectors, order $n=3$ excitation, $\alpha=1.4, \delta=1.117, \rho=1.67, \lambda=0.1$, $\gamma=(\alpha+\delta+1) / \tilde{n}^{2}, \mu_{a}=0.01, \mu_{b}=0.1, v_{a}=0.5, v_{b}=v_{c}=0.1$, $f_{\phi}=0.0016$, and $f_{\theta}=0.001$. There are two groups of $N$ natural frequencies whose spread depends primarily on the strength of the inter-sector coupling. For nonzero coupling they generally appear in repeated pairs, except for mode $p=1$ and mode $p=(N+2) / 2$ if $N$ is even. For zero coupling they collapse onto a single pair of values $[11,12]$. The bottom-most group corresponds to in-phase motions of the two DOF in each sector and the top-most curves represent out-of-phase motions of the disk/blade DOFs. The natural frequencies increase slightly with increasing rotor speed due to centrifugal stiffening.

It is well-known that e.o. excitation excites only one mode in each eigenfrequency group due to special modal orthogonality properties of cyclic systems $[11,12]$. The resonant rotor speeds can be identified in Fig. $2 a$ by the intersections of the natural frequency loci corresponding to mode $p=n+1$ with the e.o. line defined by $n \sigma .^{2}$ The corresponding resonances are shown in the frequency response diagrams of Fig. $2 b$. Of course, mis-

\footnotetext{
${ }^{2}$ The excited mode is $p=n \bmod N+1$ when considering $n \in \mathbb{Z}_{+}[12]$.
}

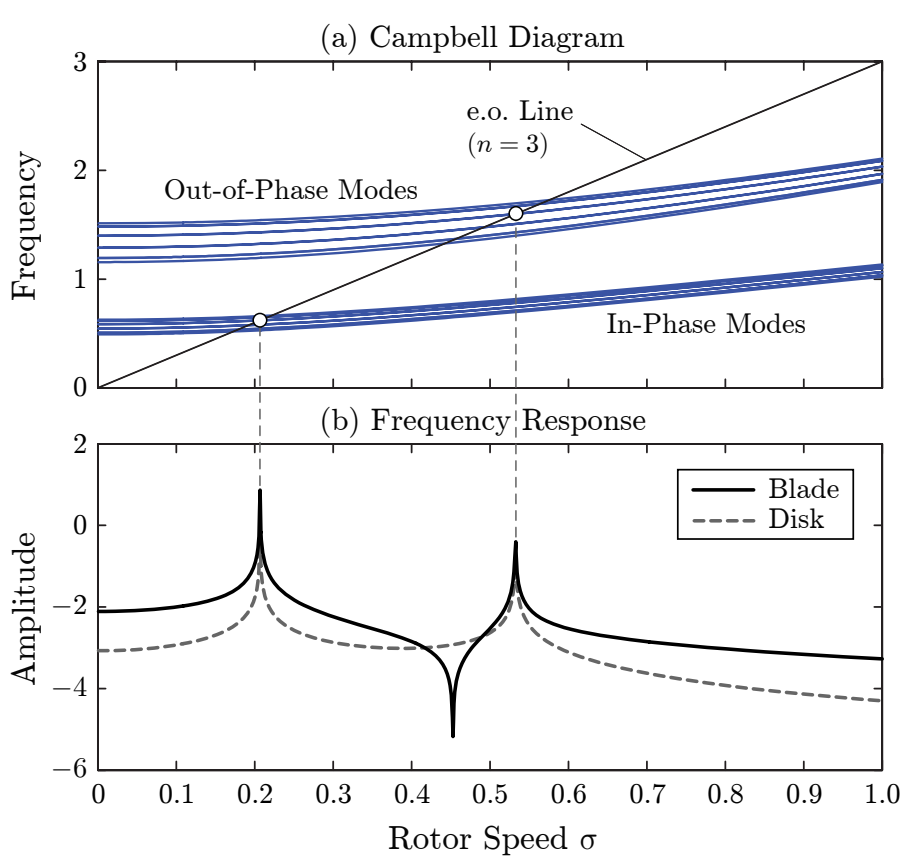

Figure 2. Campbell Diagram and Frequency Response.

tuning that arises due to imperfections among the sectors results in resonance with some degree of intensity at all of the intersections $[27,28]$.

\subsection{Response with the Absorbers Free}

When the absorbers are free to move there are three natural frequencies corresponding to each index $p \in \mathcal{N}$. The three groups of $N$ frequency loci $\bar{\omega}_{1,2,3}^{(p)}$ are plotted in Fig. 3 for increasing inter-sector coupling and increasing absorber mass while holding all other parameters fixed. Each natural frequency corresponds to a system mode, which, together with fixed intersector phase relationships, form a forward TW, backward TW, or standing wave response among the sectors, depending on the value of $n$ relative to $N[11,12,23]$. The natural frequencies are tightly-spaced for weak inter-sector coupling (Fig. 3a) and they spread out as the coupling is increased (Fig. 3c). Each group exhibits the eigenvalue veering phenomenon, or mutual repulsion of the eigenfrequencies, which results from small dynamic coupling between the disks and blades and between the blades and absorbers $[17,18,29]$. The ratio of absorber mass to the disk and blade masses is essential for the curve veering phenomenon. When the mass ratio is very small (Fig. 3d), the natural frequency curves are close to each other in the veering regions. (In Figs. $3 d-f$ only one frequency locus $\bar{\omega}_{1,2,3}^{(1)}$ is shown in each group, which corresponds to zero inter-sector coupling.) A larger difference between the groups of frequency loci can be obtained by increasing the mass ratio (Fig. $3 f$ ), although in most applications this mass ratio is small. 


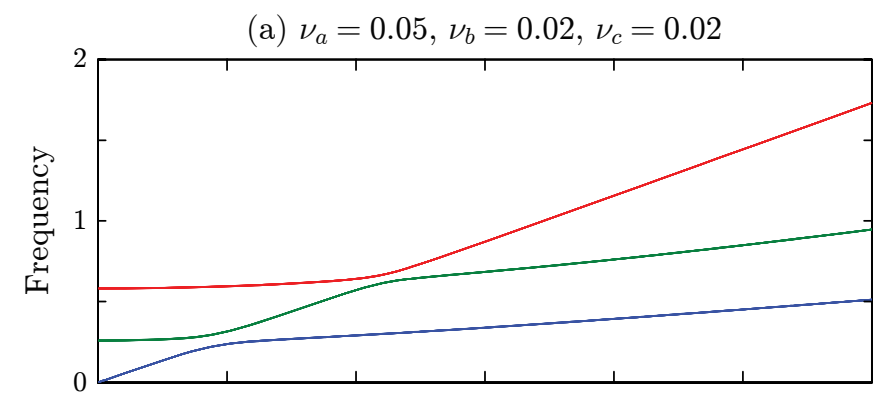

(b) $\nu_{a}=0.20, \nu_{b}=0.08, \nu_{c}=0.08$

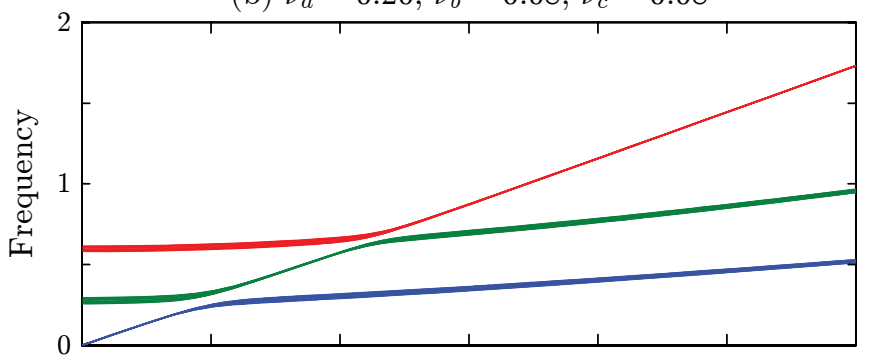

(c) $\nu_{a}=0.50, \nu_{b}=0.15, \nu_{c}=0.15$

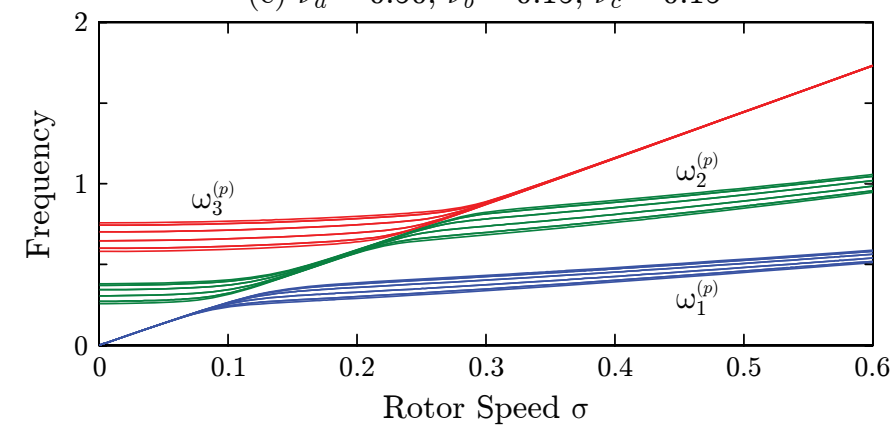

(d) $\mu_{a}=0.001$

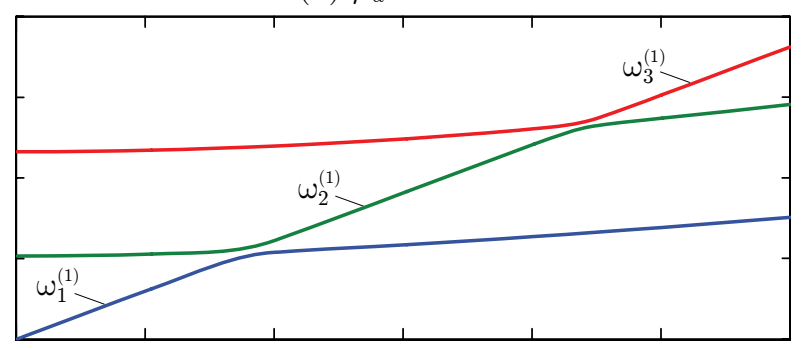

(e) $\mu_{a}=0.005$

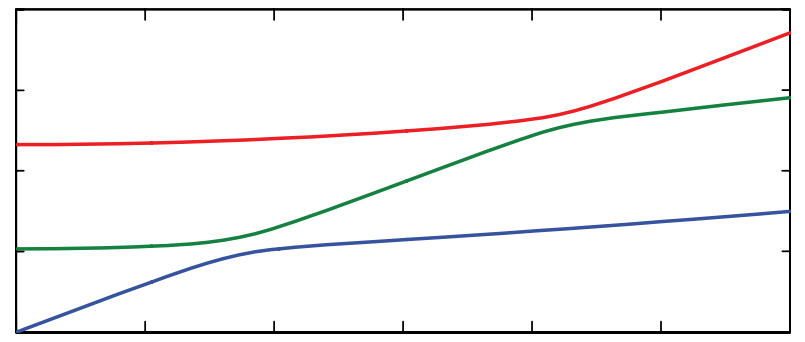

(f) $\mu_{a}=0.025$

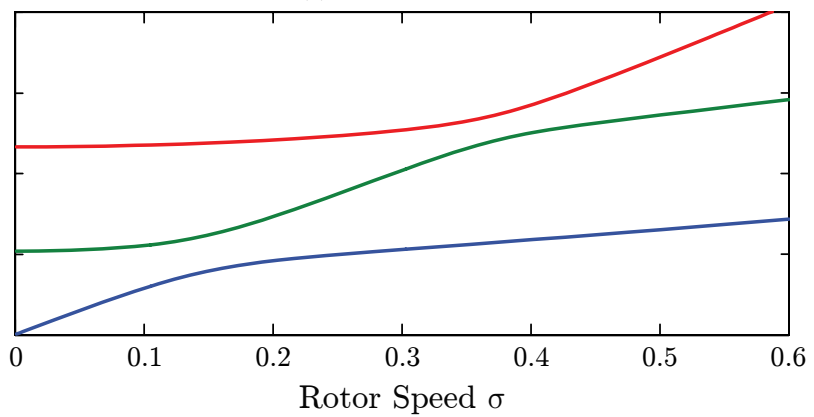

Figure 3. Campbell diagrams showing the effects of increasing inter-sector coupling and absorber mass.

Possible resonances can be identified by the intersections of $\bar{\omega}_{1,2,3}^{(p)}(\sigma)$ with the e.o. line $n \sigma$. However, there is a system resonance in the perfectly symmetric case only when

$$
n \sigma=\bar{\omega}_{1,2,3}^{(n+1)}(\sigma)
$$

is satisfied because only mode $n+1$ is excited in the steadystate $[11,12]$. The principle objectives of this work are to determine if any resonances persist for near-ideal absorber tuning (i.e., for $\beta$ close to zero); whether or not any new resonances are introduced for multi-DOF disk/blade models; and, if so, whether the absorbers can be tuned to avoid these resonances over a range of rotor speeds.

The results are summarized in Fig. 4, which shows the eigenfrequency loci in Campbell diagrams and the corresponding disk, blade, and absorber amplitude frequency responses $\left(\left|\varphi_{i}\right|,\left|\theta_{i}\right|,\left|\psi_{i}\right|\right)$ for a model with $N=10$ sectors, order $n=3$ excitation, various absorber tuning values that range from under- tuning $(\beta<0)$ to overtuning $(\beta>0)$, and for the same parameter values used in Fig. 2. Inter-sector coupling is assumed to be quite strong to more clearly show the effects of detuning on the excited modes; this does not affect the approach nor the conclusions. In many situations the eigenfrequency loci $\bar{\omega}_{1,2,3}^{(p)}$ cross the e.o. line twice. For absorber overtuning (Fig. $4 a$ ) or sufficiently large undertuning (Fig. 4b) there are two system resonances, which are indicated by circles in the Campbell diagrams. Insets 1 and 2 show close-up views of the veering regions in Fig. $4 a$, including the resonances corresponding to $\bar{\omega}_{1,2}^{(n+1)}$. Figure $4 c$ shows that the fundamental resonance disappears for ideal tuning $(\beta=0)$, but the resonance corresponding to $\bar{\omega}_{2}^{(n+1)}$ persists. In this case, there is only one system resonance over the full range of possible rotor speeds.

One of the main findings of Olson et al. is the existence of a no-resonance zone in the 2-DOF sector model dynamics, that is, a range of absorber tuning values which results in complete resonance suppression over the full range of possible rotor 
(a) $\beta=+0.1 \quad$ (Overtuning)
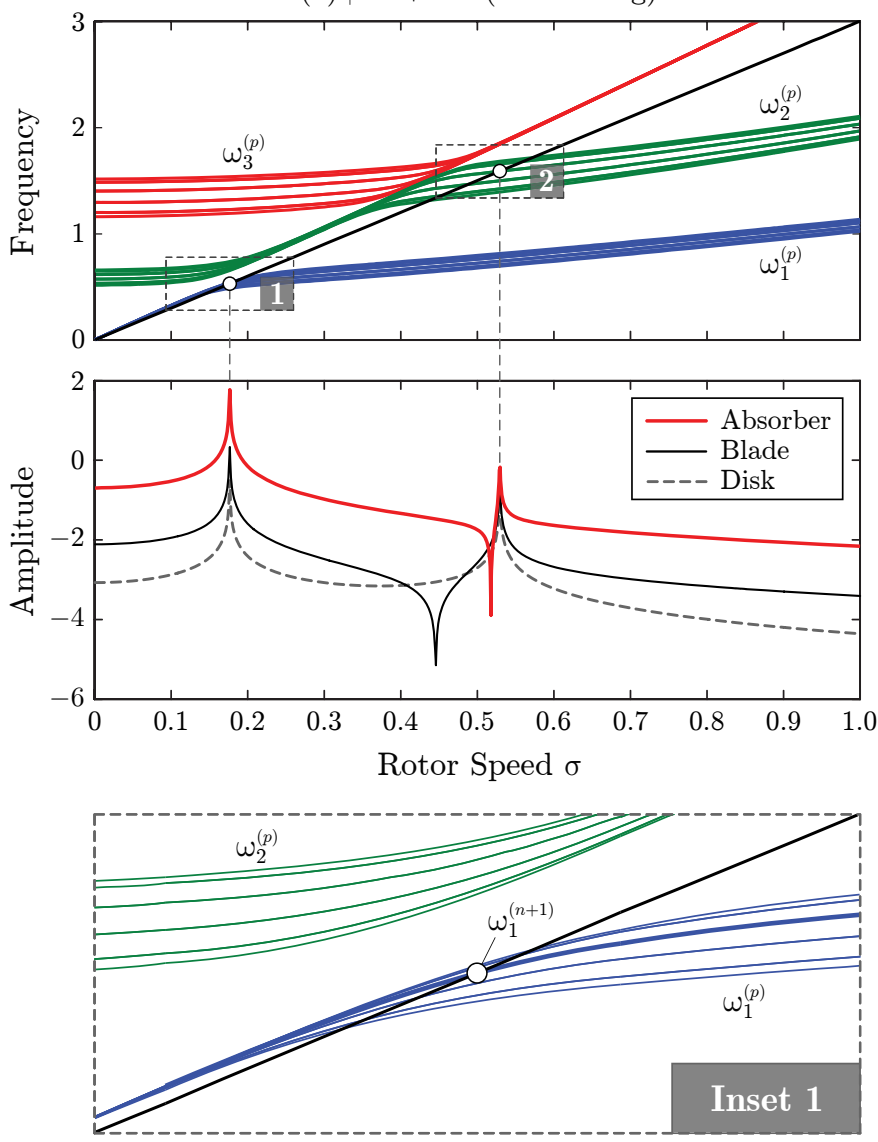

(c) $\beta=0 \quad$ (Ideal Tuning)

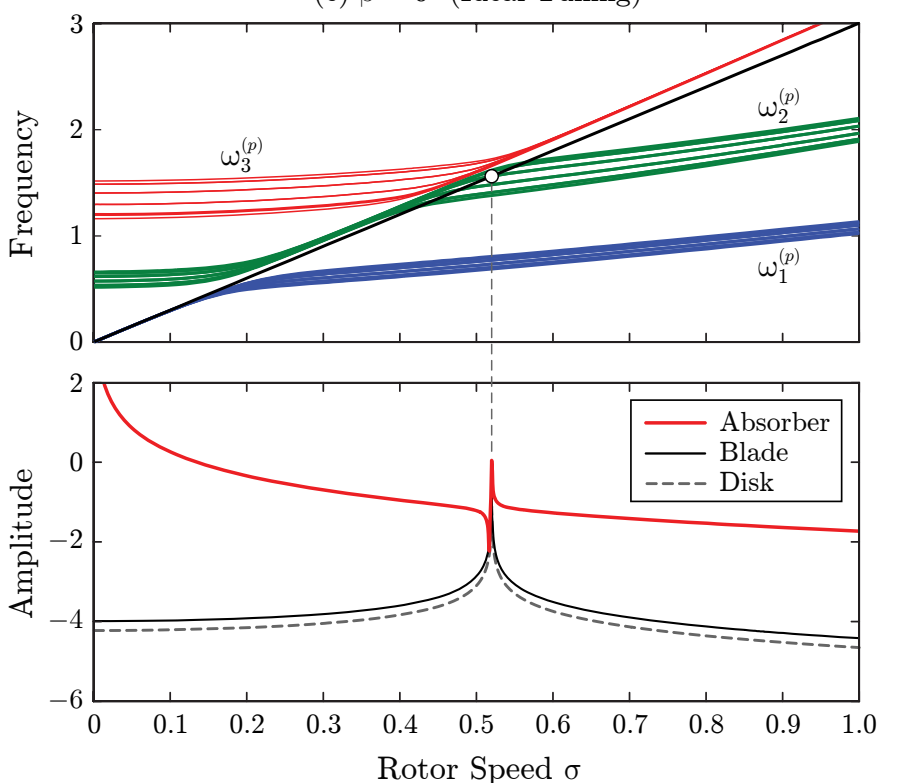

(b) $\beta=-0.1 \quad$ (Undertuning)
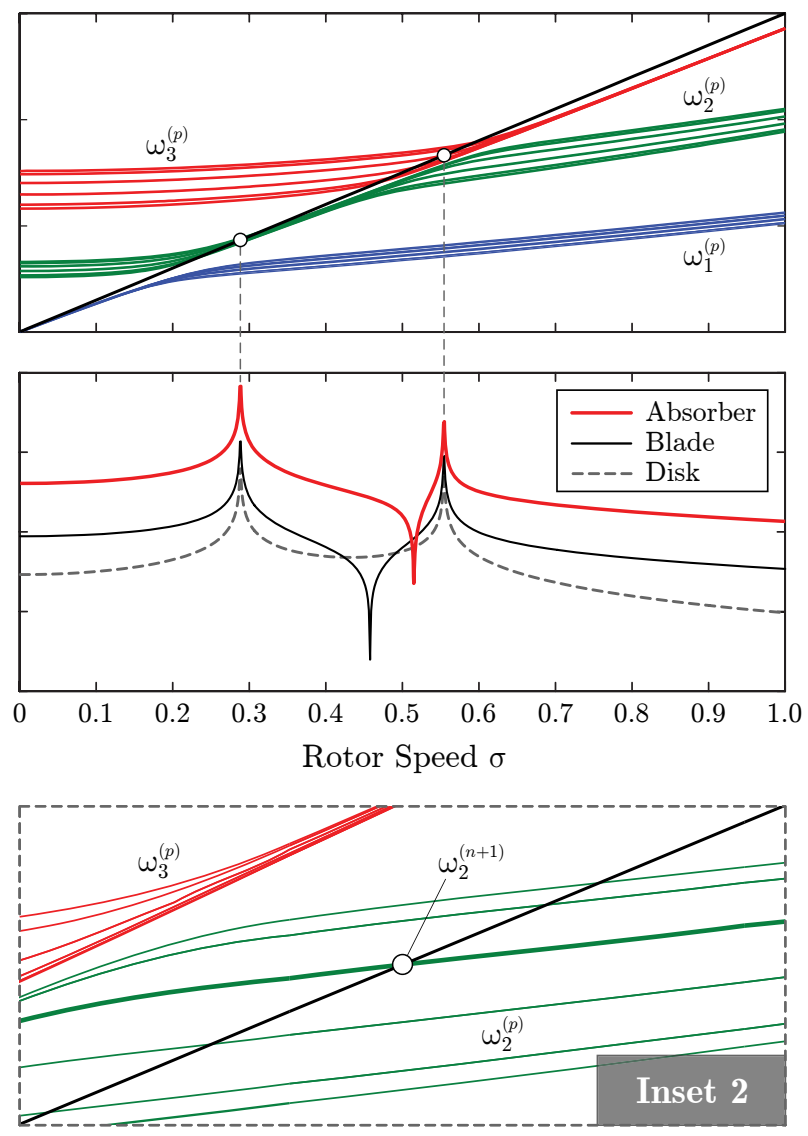

(d) $\beta=-0.035 \quad$ (Slight Undertuning)
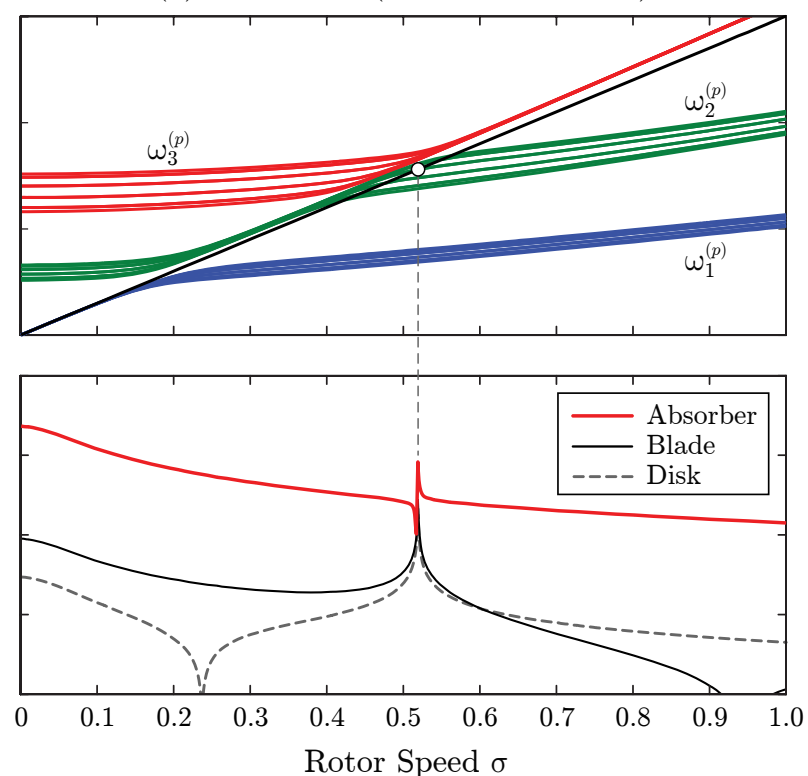

Figure 4. Campbell diagrams and the corresponding amplitude frequency response. 


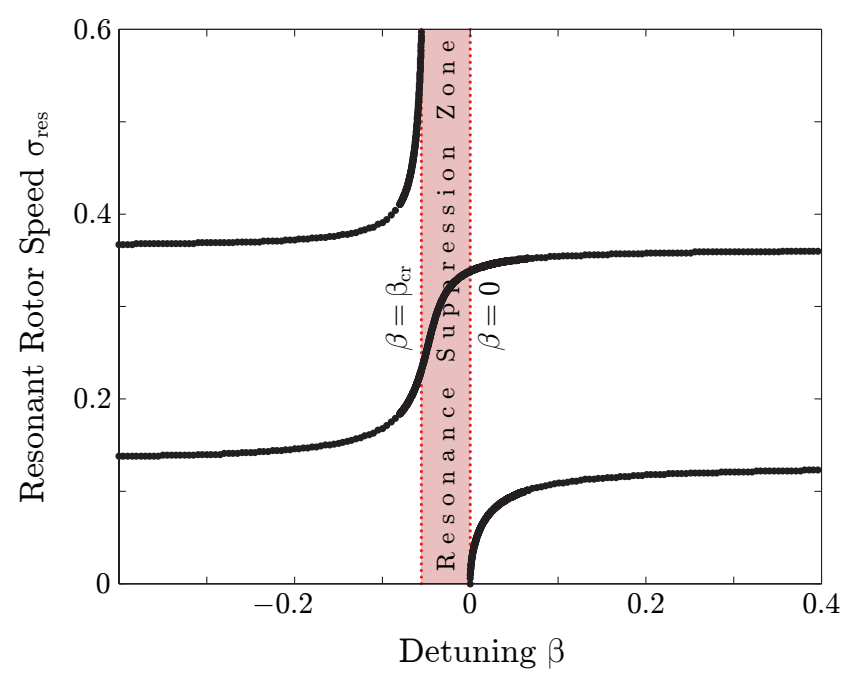

Figure 5. Resonant Rotor Speed Versus Detuning.

speeds $[11,12]$. The no-resonance gap corresponds to a finite range of absorber undertuning values, where the critical lower bound $\beta_{\mathrm{cr}}<0$ depends on system and absorber parameters and the upper bound corresponds to ideal tuning with $\beta=0$. Robust absorber design involves intentional undertuning within this (generally small) range, which allows for small variations of the model or parameter values without introducing resonance. For the 3-DOF sector model shown in Fig. $1 b$ the no-resonance zone is replaced by a resonance suppression zone defined by

$$
\beta_{\mathrm{cr}}<\beta \leq 0
$$

where one of two resonances is avoided but the other resonance persists. It is clear from the eigenfrequency structures shown in Fig. 4 that there will be $P-1$ excited modes for the general case of $P \geq 2$ disk/blade DOFs per sector.

The resonance suppression zone can be clearly identified in Fig. 5, which is a plot of the resonant rotor speed $\sigma_{\text {res }}$ (defined implicitly by Eq. (9)) in terms of the detuning parameter $\beta$ for the same parameter values used in Fig. 2. For the case of $P=2$ DOFs per sector considered by Olson et al. [11-16] there exists a range of undertuning values for which there are no system resonances (i.e., the no-resonance zone). Figure 5 shows that no such range exists for $P=3$ DOFs per sector, and it is clear that the same can be said for the general case of $P \geq 3$. Instead, there is a finite range defined by Eq. (10) for which one resonance is robustly avoided and one resonance persists. For the parameter values used in Fig. 4 the critical undertuning value is $\beta_{\mathrm{cr}}=-0.0554$. Fig. $4 d$ shows example disk, blade, and absorber frequency response plots and the corresponding Campbell diagram for tuning within the resonance suppression zone.

\section{CONCLUSIONS AND DIRECTIONS FOR FUTURE WORK}

The performance of centrifugally-driven, order-tuned vibration absorbers has been investigated using a higher-fidelity lumped-parameter model of a bladed disk assembly under e.o. excitation. The model consists of a cyclic array of $N$ identical, identically-coupled sectors, each with three DOFs that capture the effects of the disk, blades, and absorbers. The principle finding of this work is the existence of an absorber undertuning region in which one resonance is robustly avoided. The range is bounded from above by ideal tuning and from below by a critical linear undertuning, which forms a resonance-suppression zone. Thus, while the absolute no-resonance zone reported by Olson et al. [11-13] does not extend completely to higher-fidelity sector models, the absorbers do provide a range of speeds over which one resonance can be suppressed.

It is important to note that adding more DOFs per sector does not qualitatively alter this picture. That is, groups of higherorder modes will still experience resonance, albeit at higher rotor speeds. This is due to the structure of the natural frequency curves shown in Fig. 4, which veer only once and then flatten out (asymptotically approach the absorbers-locked frequencies $[11,12])$ as the rotor speed is increased. Therefore, the e.o. line generically crosses all but one natural frequency group. Adding more DOFs per sector simply adds more such curves at higher frequencies, thereby introducing more resonances at higher rotor speeds. The main conclusion is that an identical set of ordertuned absorbers can suppress only one resonance.

There remains much work to be done on this class of problems, which can be divided into four general categories. First, very few systematic experiments have been performed. The work by Duffy and co-workers on self-tuned impact dampers makes use of an impacting mode of motion for order-tuned absorbers, and demonstrates that they are effective in attenuating vibrations in a rotating plate [30,31].

The second group of problems address nonlinear effects. Shaw and Pierre [16] have investigated a single-sector model with an order-tuned absorber that undergoes impacts when it reaches a certain amplitude. Olson and Shaw [12, 14, 15] analyzed the response of a two-DOF-per-sector model in which absorber path nonlinearity is taken into account, and they make some recommendations about nonlinear absorber tuning. Specifically, the absorber path, which is generalized to be non-circular, should be slightly softening for large amplitudes. Polchi [19] has some preliminary results along the same lines for the threeDOF-per-sector model considered here, which show that the linear resonance structure essentially persists for weakly nonlinear absorber motions. However, a detailed analysis with recommendations for path design remains to be completed.

The third category deals with systems of multiple absorbers, for which there are many possible studies. For example, is it necessary to have an absorber in every sector? The answer to 
this question likely depends on the level of intersector coupling. Also, can one use sets of absorbers to address multiple resonances? An affirmative answer to this question is given for the case of a single sector by Wang et. al [32], but the multi-sector case has yet to be considered. The question of whether or not sets of absorbers can be arranged so that not every sector has absorbers tuned to every targeted order should also be considered.

The final, and probably most practical, line of work should consider the effects of mistuning and intentional detuning of the primary system and absorbers. It is well known that slight mistuning of cyclic systems leads to localization of vibration modes and of the forced steady-state response [33-36]. All of the issues introduced by mistuning exist, and indeed are compounded, by the presence of absorbers, which raises a number questions. If the absorbers and/or sectors have mistuning, what is the degree of localization in the overall response? Of course, localized absorber motions are highly undesirable because the absorbers have only limited rattle space in which to operate. So, can one intentionally detune the system (sectors and/or absorbers) to mitigate the localization? Will such detuning be random, or just of some order of symmetry less than that of the ideal cyclic system? These questions, and no doubt others, should be addressed to more fully understand the effectiveness of order-tuned absorbers in practical systems.

\section{ACKNOWLEDGMENT}

The authors are grateful to Mathias Legrand for many enlightening discussions related to this work. This work was supported by the National Science Foundation (NSF) under grant NSF-0408866. Any opinions, findings and conclusions or recommendations expressed in this material are those of the authors and do not necessarily reflect the views of the NSF.

\section{REFERENCES}

[1] Alsuwaiyan, A. S., 1999. "Performance stability and localization of systems of vibration absorbers". Ph.D. dissertation, Michigan State University, East Lansing, MI.

[2] Haddow, A. G., and Shaw, S. W., 2003. "Centrifugal Pendulum Vibration Absorbers: An Experimental and Theoretical Investigation”. Nonlinear Dynamics, 34, pp. 293-307.

[3] Alsuwaiyan, A. S., and Shaw, S. W., 2002. "Performance and Dynamic Stability of General-Path Centrifugal Pendulum Vibration Absorbers". Journal of Sound and Vibration, 252(5), pp. 791-815.

[4] Alsuwaiyan, A. S., and Shaw, S. W., 1999. "Localization of Free Vibration Modes in Systems of Nearly-Identical Vibration Absorbers". Journal of Sound and Vibration, 228(3), pp. 703-711.

[5] Lee, C. T., and Shaw, S. W., 1996. "On the Counteraction of Periodic Torques in Rotating Systems Using Centrifu- gally Driven Vibration Absorbers". Journal of Sound and Vibration, 191(5), pp. 695-719.

[6] Alsuwaiyan, A. S., and Shaw, S. W., 2003. "SteadyState Response of Systems of Nearly-Identical Torsional Vibration Absorbers". Journal of Vibration and Acoustics, 125(1), pp. 80-87.

[7] Ker Wilson, W., 1968. Practical Solutions of Torsional Vibration Problems, 3rd ed., Vol. IV. Champman and Hall Ltd, London, ch. XXX.

[8] Wachs, M., 1973. “The Main Rotor Bifilar Absorber and its Effect on Helicopter Reliability/Maintainability”. SAE Technical Paper Series 730894.

[9] Cook, M., 1994. "Absolute Absorbtion”. Car Craft Magazine, August, p. 75.

[10] Nester, T. M., Haddow, A. G., Shaw, S. W., Brevick, J. E., and Borowski., V. J., 2003. "Vibration Reduction in Variable Displacement Engines Using Pendulum Absorbers". In Proceedings of the SAE Noise and Vibration Conference and Exhibition, no. 2003-01-1484.

[11] Olson, B. J., Shaw, S. W., and Pierre, C. "Vibration absorbers for a rotating flexible structure with cyclic symmetry: Linear order tuning". in review.

[12] Olson, B. J., 2006. "Order-Tuned Vibration Absorbers for Systems with Cyclic Symmetry with Applications to Turbomachinery". Ph.D. dissertation, Michigan State University, East Lansing, MI.

[13] Olson, B. J., Shaw, S. W., and Pierre, C., 2005. "OrderTuned Vibration Absorbers for Cyclic Rotating Flexible Structures". In Proceedings of the 2005 ASME Design Engineering Technical Conferences, 20th Biennial Conference on Mechanical Vibration and Noise, no. DETC200584641.

[14] Olson, B. J., and Shaw, S. W. "Vibration absorbers for a rotating flexible structure with cyclic symmetry: Nonlinear path design". to appear.

[15] Olson, B. J., and Shaw, S. W., 2008. "Vibration absorbers for cyclic rotating flexible structures: Linear and nonlinear tuning". In Proceedings of the 2008 ASME Conference on Smart Materials, Adaptive Structures and Intelligent Systems, no. SMASIS08-632.

[16] Shaw, S. W., and Pierre, C., 2006. "The Dynamic Response of Tuned Impact Absorbers for Rotating Flexible Structures". Journal of Computational and Nonlinear Dynamics, 1, pp. 13-24.

[17] Pierre, C., 1988. "Mode Localization and Eigenvalue Loci Veering Phenomena in Disordered Structures". Journal of Sound and Vibration, 126(3), pp. 485-502.

[18] Happawana, G. S., Bajaj, A. K., and Nwokah, O. D. I., 1993. "A singular perturbation analysis of eigenvalue veering and modal sensitivity in perturbed linear periodic systems". Journal of Sound and Vibration, 160(2), pp. 225242. 
[19] Polchi, S., 2007. Multi-scaling and averaging for higher fidelity lumped model of bladed disk with cpva. Tech. rep., McGill University.

[20] Ewins, D. J., 1973. "Vibration Characteristics of Bladed Disc Assemblies". Journal of Mechanical Engineering Science, 15(3), pp. 165-186.

[21] Den Hartog, J. P., 1938. "Tuned Pendulums as Torsional Vibration Eliminators". In Stephen Timoshenko 60th Anniversary Volume. The Macmillan Company, New York, pp. 1726.

[22] Meirovitch, L., 1997. Principles and Techniques of Vibrations. Prentice Hall, Upper Saddle River.

[23] Óttarsson, G. S., 1994. "Dynamic Modeling and Vibration Analysis of Mistuned Bladed Disks". Ph.D. dissertation, University of Michigan, Ann Arbor, MI.

[24] Wagner, L. F., and Griffin, J. H., 1996. "Forced Harmonic Response of Grouped Blade Systems: Part I-Discrete Theory". Journal of Engineering for Gas Turbines and Power, 118, pp. 130-136.

[25] Cai, C. W., Cheung, Y. K., and Chan, H. C., 1990. "Uncoupling of Dynamic Equations for Periodic Structures". Journal of Sound and Vibration, 139(2), pp. 253-263.

[26] Shen, I. Y., 1994. "Vibration of Rotationally Periodic Structures". Journal of Sound and Vibration, 172(4), pp. 459470.

[27] Wei, S. T., and Pierre, C., 1988. "Localization Phenomena in Mistuned Assemblies with Cyclic Symmetry Part I: Free Vibrations". Journal of Vibration, Acoustics, Stress, and Reliability in Design, 110, pp. 429-438.

[28] Wei, S. T., and Pierre, C., 1988. "Localization Phenomena in Mistuned Assemblies with Cyclic Symmetry Part II: Forced Vibrations". Journal of Vibration, Acoustics, Stress, and Reliability in Design, 110, pp. 439-449.

[29] Perkins, N. C., and C. D. Mode, J., 1986. "Comments on Curve Veering in Eigenvalue Problems". Journal of Sound and Vibration, 106(3), pp. 451-463.

[30] Duffy, K. P., Bagley, R. L., and Mehmed, O., 2000. “On a Self-Tuning Impact Vibration Damper for Rotating Turbomachinery". In 36th AIAA/ASME/SAE/ASEE Joint Propulsion Conference and Exhibit, no. AIAA-2000-3100.

[31] Duffy, K., Mehmed, O., and Johnson, D., 2001. "SelfTuning Impact Dampers for Fan and Turbine Blades". In Proceedings of the 6th National Turbine Engine High Cycle Fatigue (HCF) Conference.

[32] Wang, Y., Chao, C. P., and Shaw, S. W., 1997. "Design of Pendulum Vibration Absorbers for the Attenuation of Transverse Vibrations in Rotating Beams". In Proceedings of the ASME 17th Biennial Conference on Mechanical Vibration and Noise, no. DETC97/VIB-4182.

[33] Castanier, M. P., and Pierre, C., 2002. "Using Intentional Mistuning in the Design of Turbomachinery Rotors". AIAA Journal, 40(10), pp. 2077-2086.
[34] Castanier, M. P., and Pierre, C., 1997. "Consideration on the Benefits of Intentional Blade Mistuning for the Forced Response of Turbomachinery Rotors". Analysis and Design Issues for Modern Aerospace Vehicles, 55, pp. 419-425.

[35] Whitehead, D. S., 1966. "Effect of Mistuning on the Vibration of Turbomachine Blades Induced by Wakes". Journal of Mechanical Engineering Science, 8(1), pp. 15-21.

[36] Ewins, D. J., 1969. "The Effect of Detuning upon the Forced Vibrations of Bladed Disks". Journal of Sound and Vibration, 9(1), pp. 65-79. 